\title{
Assessing calvarial vault constriction associated with helmet therapy in deformational plagiocephaly
}

\author{
Erin C. Peterson, BS, ${ }^{1}$ Kamlesh B. Patel, MD, MSc, ${ }^{1}$ Gary B. Skolnick, BS, ${ }^{1}$ Kristin D. Pfeifauf, JD, ${ }^{1}$ \\ Katelyn N. Davidson, ${ }^{2}$ Matthew D. Smyth, MD, ${ }^{3}$ and Sybill D. Naidoo, PhD, RN, CPNP1
}

'Department of Surgery, Division of Plastic and Reconstructive Surgery, Washington University School of Medicine in St. Louis, Missouri; 'Boston College, Chestnut Hill, Massachusetts; and ${ }^{3}$ Departments of Surgery and Neurosurgery, Division of Pediatric Neurosurgery, Washington University School of Medicine in St. Louis, Missouri

\begin{abstract}
OBJECTIVE Deformational plagiocephaly and/or brachycephaly (DPB) is a cranial flattening frequently treated in pediatric craniofacial centers. The standard of care for DPB involves patient positioning or helmet therapy. Orthotic therapy successfully reduces cranial asymmetry, but there is concern over whether the orthotics have the potential to restrict cranial growth. Previous research addressing helmet safety was limited by lack of volume measurements and serial data. The purpose of this study was to directly compare head growth data in patients with DPB between those who underwent helmet therapy and those who received repositioning therapy.
\end{abstract}

METHODS This retrospective cohort study analyzed pre- and posttherapy 3D photographs of 57 patients with DPB who had helmet therapy and a control group of 57 patients with DPB who underwent repositioning therapy. The authors determined the change in cranial vault volume and cranial circumference between each patient's photographs using 3D photogrammetry. They also computed a cubic volume calculated by multiplying anterior-posterior diameter, biparietal diameter, and height. Linear regressions were used to quantify effects of age and therapy type on these quantities.

RESULTS A comparison of the following variables between the two groups yielded nonsignificant results: age at the beginning $(p=0.861)$ and end $(p=0.539)$ of therapy, therapy duration $(p=0.161)$, and the ratio of males to females $(p=$ 0.689 ). There was no significant difference between patients who underwent helmeting versus positioning therapy with respect to change in either volume calculation or head circumference z-score $(p \geq 0.545)$. Pretherapy photograph age was a significant predictor of cranial growth ( $p \leq 0.001)$, but therapy type was not predictive of the change in the study measurements $(p \leq 0.210)$.

CONCLUSIONS The authors found no evidence that helmet therapy was associated with cranial constriction in the study population of patients with DPB. These results strengthen previous research supporting helmet safety and should allow health care providers and families to choose the appropriate therapy without concern for potential negative effects on cranial growth.

https://thejns.org/doi/abs/10.3171/2018.2.PEDS17634

KEYWORDS deformational plagiocephaly; helmet therapy; cranial constriction; craniofacial

$\mathrm{D}$ EFORMATIONAL plagiocephaly is a nonsynostotic skull asymmetry, with a current prevalence of nearly $20 \%$ at 4 months of age. . $^{2,8,31,33}$ The skull shape resembles a parallelogram and results from external pressures that flatten the lateral occiput, and it can produce an anterior shift of the ipsilateral ear and ipsilateral frontal bossing. ${ }^{10,12,16,24}$ Brachycephaly is a related condition involving a more symmetrical flattening of the occiput., 43 The 1992 American Academy of Pediatrics' "Back to Sleep" program ${ }^{11}$ successfully reduced the rate of sudden infant death syndrome by advocating for infants to sleep in the supine position, but since its implementation the prevalence of deformational plagiocephaly has risen substantially. ${ }^{10,12,16,21}$ This suggests a causative relationship

ABBREVIATIONS CVV = cranial vault volume; DPB = deformational plagiocephaly and/or brachycephaly; ICC = intraclass correlation coefficient.

SUBMITTED November 14, 2017. ACCEPTED February 19, 2018

INCLUDE WHEN CITING Published online May 11, 2018; DOI: 10.3171/2018.2.PEDS17634 
between supine position and deformational plagiocephaly. $5,8,10,19,21$ Studies have also found an association between deformational plagiocephaly and developmental delay, but it is most likely that both conditions arise from a separate common etiology., 37,28 The current standard of care for deformational plagiocephaly and/or brachycephaly (DPB) involves active repositioning or orthotic helmeting.7.12, 16,22 Recent systematic analyses found helmeting often reduces cranial asymmetry faster than positioning. ${ }^{25,32,34}$ Positioning therapy involves laying the child down in a way that avoids pressure on the flattened area and additional supervised time on their stomach. ${ }^{24,25}$ Helmeting is started between 4 and 6 months of age, is worn for 22-23 hours per day for a duration of 3-6 months, and is used for moderate to severe deformations. ${ }^{12,13,23}$ Known risks of helmeting are few and rare, with skin irritation being the most common. $7,13,19,25$ Helmet therapy is concluded when the skull reaches normal anthropometric measurements or when parents are satisfied with the aesthetic outcome of the reshaping.

The research on DPB patients has largely focused on one- and two-dimension anthropometric measurements, ${ }^{16,22,}$ $25,29,30$ rather than overall cranial volume. Our study utilizes serial 3D photogrammetry to measure the change in calvarial vault circumference and volume before and after DPB helmet therapy to assess potential calvarial vault constriction. This study aims to further the understanding of the safety and effects of helmet therapy in DPB patients.

\section{Methods}

A retrospective cohort study of patients with DPB was performed following IRB approval. All patients were diagnosed at a single pediatric plastic surgery DPB clinic between 2004 and 2013. Therapy choice was arrived at by a mutual decision between the child's guardians and a single medical provider (S.D.N.). This decision involved considerations including aesthetic concerns, severity of the DPB, costs, and family accessibility to additional appointments for helmet fittings and adjustments. Patient data such as age, diagnosis, and the type and timing of therapy were culled from the Craniofacial Anomalies Archive at our institution. ${ }^{26}$ Inclusion criteria were diagnosis of DPB at or before 6 months of age and high-quality preand posttherapy $3 \mathrm{D}$ photographs taken before 12 months of age. Patients who were syndromic or had other craniofacial malformations and those with a therapy duration of fewer than 30 days were excluded. Figure 1 graphically depicts the study selection process. Fifty-seven repositioning therapy patients met the inclusion criteria. Fifty-seven

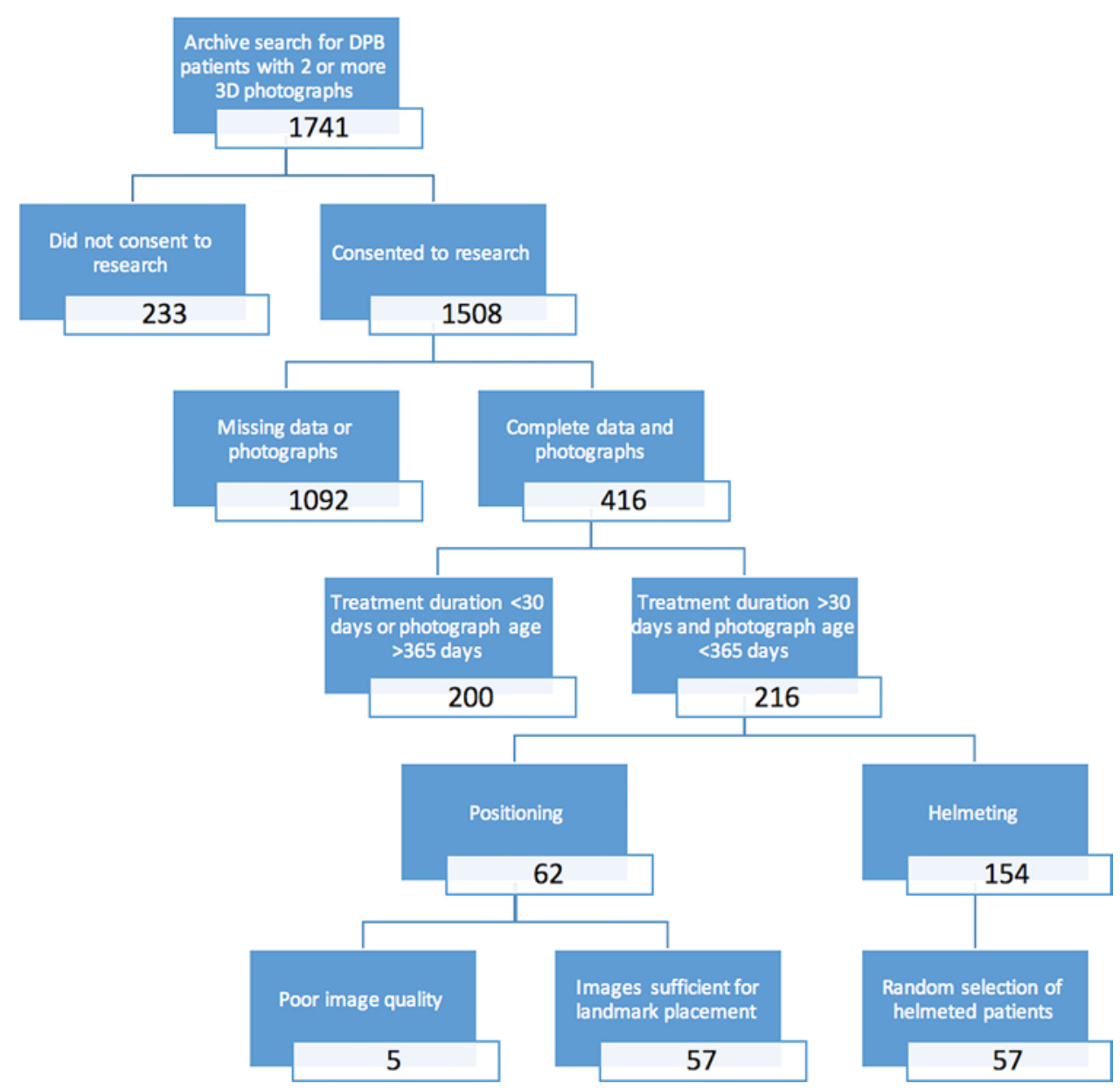

FIG. 1. The patient selection process from the Craniofacial Anomalies Archive. Figure is available in color online only. 
of the 154 possible helmet therapy candidates were selected to create an equal comparison group with regard to age at pretreatment photography and to treatment duration.

Serial photographs were taken by a 4-pod Cranial System camera from $3 \mathrm{dMD}$. Image analysis was performed with 3dMD Vultus v. 2.2.024. Measurements were taken following orientation to the Frankfort horizontal (Fig. 2). Study measurements included anteroposterior diameter, biparietal diameter, height, circumference, and cranial vault volume (CVV) as previously described ${ }^{14}$ by Le et al. As described, the CVV was defined as the volume cranial to the plane aligned with the external acoustic meatus and the lateral epicanthus and was adjusted using the equation developed by McKay et al. ${ }^{20}$ Additionally we multiplied anteroposterior diameter, biparietal diameter, and height to generate a cubic volume. Circumference measurements were converted to z-scores using the CDC/NCHS Infant Head Circumference for Age Percentiles ( $<36$ months) calculator (https://reference.medscape.com/calculator/infanthead-circumference).

Photographs that did not completely capture the superior cranial region required additional processing to calculate CVV. These images were converted to stl file format and then imported into Blender v. 2.78 (www.blender.org; programmer Michael Itagaki, 2015). The imported objects were encapsulated in a spherical mesh. The shrink-wrap tool was used to fill any holes in the object mesh. The CVV was calculated from this shrink-wrapped volume (Fig. 3). To assess the validity of this method, one photograph with an intact vertex was shrink-wrapped. The meshes had similar circumferences and volume measurements before and after shrink-wrapping. We then shrinkwrapped a photograph with a hole at the superior end of the calvaria. The meshes had similar circumferences

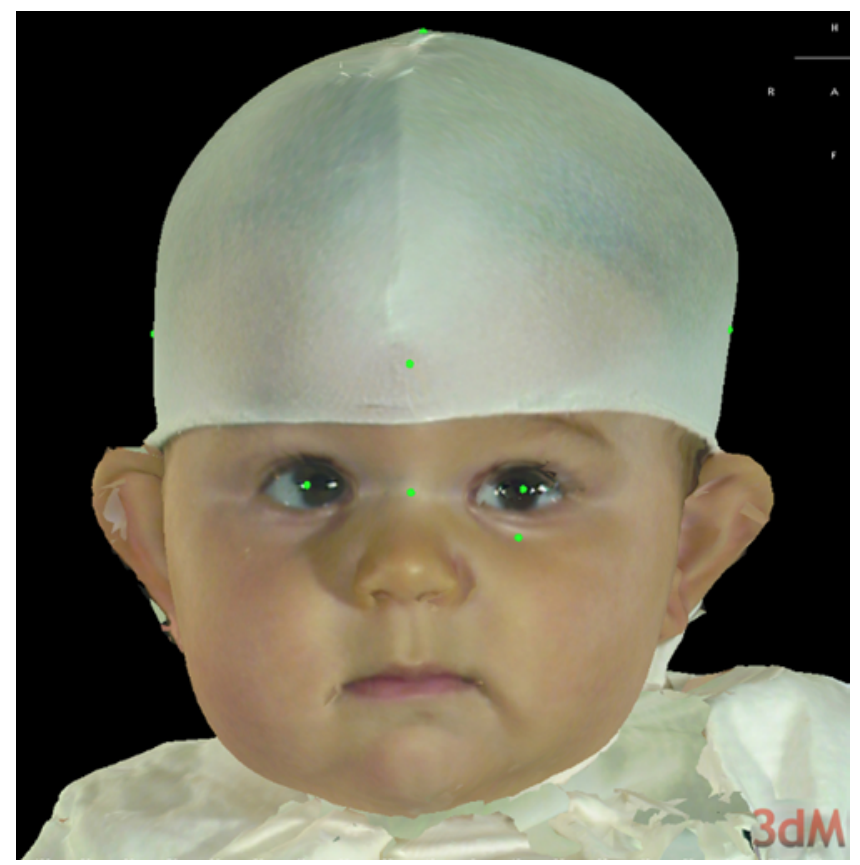

FIG. 2. Three-dimensional photograph of infant with green landmarks placed for study measurements. Figure is available in color online only.

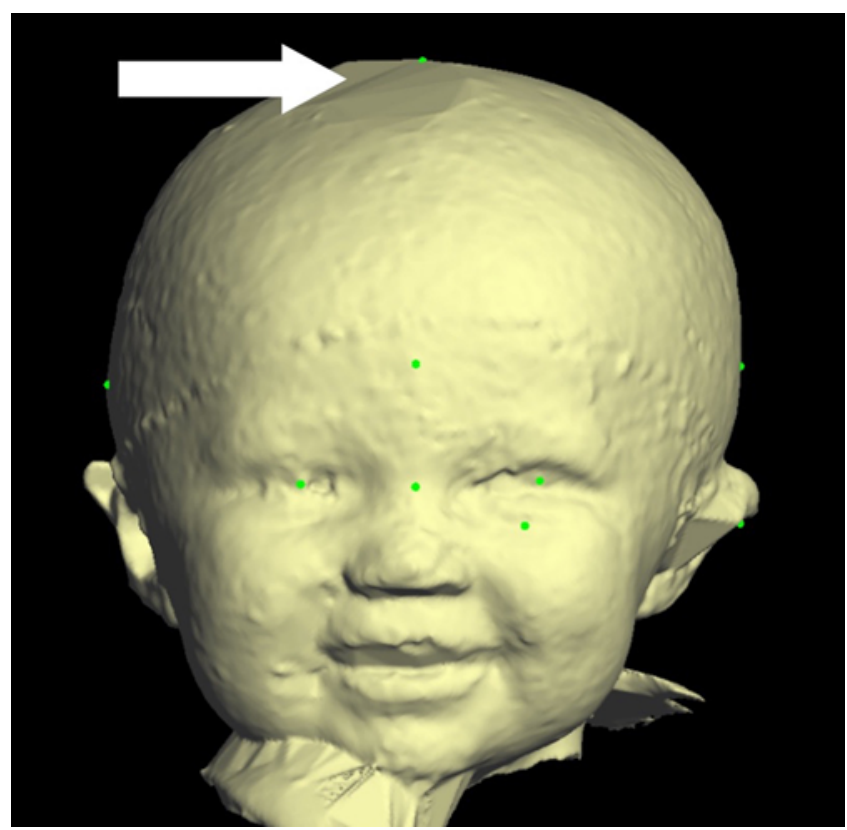

FIG. 3. Three-dimensional photograph wrapped with Blender software with green landmarks placed for study measurements. Note the arrow indicating slightly flattened area where the wrapping feature filled in a hole at the vertex. Figure is available in color online only.

before and after wrapping, but wrapping increased the CVV by $200 \mathrm{ml}$. These results confirmed that the shrinkwrapped meshes more accurately represent the CVV of the flawed photographs, allowing for comparisons with the intact head images.

A single researcher (E.C.P.) obtained all study measurements to maintain landmark placement consistency. A reliability test was performed by measuring the circumference and volume of 15 3D photographs on two separate occasions. This method produced strong reliability, with a single-measure intraclass correlation coefficient (ICC) of 0.966 ( $n=15$; CI 0.903-0.989) for circumference measurements and an ICC of 0.998 ( $n=15$; CI 0.994-0.999) for volume measurements. A power analysis (power level 0.8 , significance level 0.05 ) calculated the ability to detect a daily mean volume change of $0.32 \pm 0.005 \mathrm{ml}( \pm \mathrm{SD})$ (given treatment duration 110 days).

We used SPSS (IBM SPSS Statistics for Windows, V. 23.0) for data analysis. The total changes between the preand posttherapy photographs, as well as the average daily changes in these values, were calculated. Extreme outliers identified using Tukey's test with a multiplier of 3 were excluded from reported results. Independent-sample twotailed Student t-tests were used to compare the two therapy groups. Additionally, linear regressions were run for daily change in cubic volume (anteroposterior diameter $x$ biparietal diameter $\times$ height) and CVV using pretherapy photograph age as a covariate. Proportions of categorical variables were compared using Fisher's exact test. Statistical significance was set at $\mathrm{p}<0.05$.

\section{Results}

We analyzed a total of 224 3D photographs for this 
TABLE 1. Patient demographics

\begin{tabular}{lccc}
\hline \multirow{2}{*}{ Demographic Data } & \multicolumn{2}{c}{ Therapy } & $p$ \\
\cline { 2 - 3 } & Positioning & Helmeting & Value \\
\hline No. of patients & 57 & 57 & \\
\hline Male/female $(\% / \%)$ & $40 / 17(70 / 30)$ & $37 / 20(65 / 35)$ & 0.689 \\
\hline Race $(\%)$ & & & \\
\hline$\quad$ Caucasian & $53(93)$ & $53(93)$ & 0.818 \\
\hline$\quad$ African American & $2(4)$ & $3(5)$ & \\
\hline$\quad$ Other & $2(4)$ & $1(2)$ & \\
\hline $\begin{array}{l}\text { Pretherapy photograph age, mos } \\
\text { Posttherapy photograph age, } \\
\quad \text { mos }\end{array}$ & $4.6 \pm 1.7$ & $4.5 \pm 0.9$ & 0.861 \\
\hline Therapy duration, mos & $8.4 \pm 2.0$ & $8.6 \pm 1.4$ & 0.539 \\
\hline
\end{tabular}

Data are presented as a number, ratio $(\%)$, count $(\%)$, or mean \pm SD.

study (one pretherapy and one posttherapy for each patient). Patient demographics were similar between the two therapy groups, as seen in Table 1 . The mean therapy duration was 3.9 months for patients who underwent positioning therapy and 3.4 months for those who underwent helmet therapy.

There was no statistical significance between the aver- age change or the average daily change in the two groups' circumference $\mathrm{z}$-score, $\mathrm{CVV}$, and cubic volume values ( $\mathrm{p}$ $\geq 0.545$ ). Figure 4 displays the data for the daily changes in the CVV (Fig. 4A), cubic volume (Fig. 4B), and the overall change in circumference z-scores (Fig. 4C).

Graphic representation of the data suggested a strong correlation between age at initiation of therapy and rate of growth (Fig. 5). The results of linear regressions, to take this into account, are presented in Table 2. Age at pretherapy photograph significantly predicted the change in CVV and cubic volume. Therapy type did not significantly impact either measure.

\section{Discussion}

Deformational plagiocephaly and/or brachycephaly is a commonly treated condition by pediatric craniofacial providers. ${ }^{1,2,4,5}$ Currently there are two main therapy options for these patients: positioning of the patient and wearing a helmet. ${ }^{7,12,16,22}$ Infant age at initiation of therapy, severity of the deformation, and compliance with the positioning or helmeting guidelines can influence therapy results..$^{19}$ In repositioning therapy, the parents are educated on how to lay the infant in a way that avoids pressure on the flattened area. This includes spending less time in car seats, swings, and other seating or carrier devices where
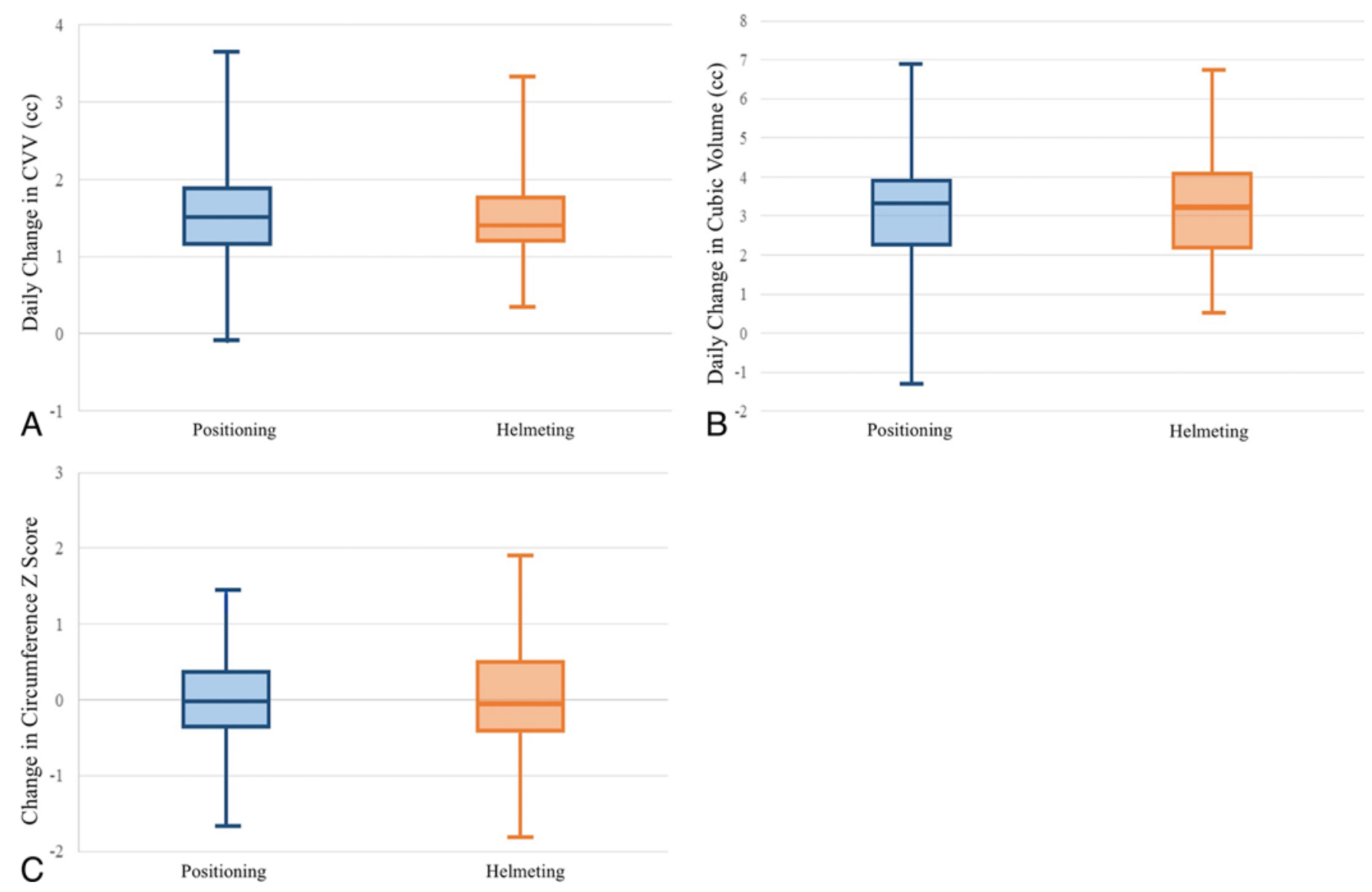

FIG. 4. A comparison of the daily change in CVV (A) and cubic volume (B), and the total change in circumference z-scores (C) between the two therapy groups. From top to bottom, horizontal lines of the boxplots represent maximum, upper quartile, median, lower quartile, and minimum. Figure is available in color online only. 


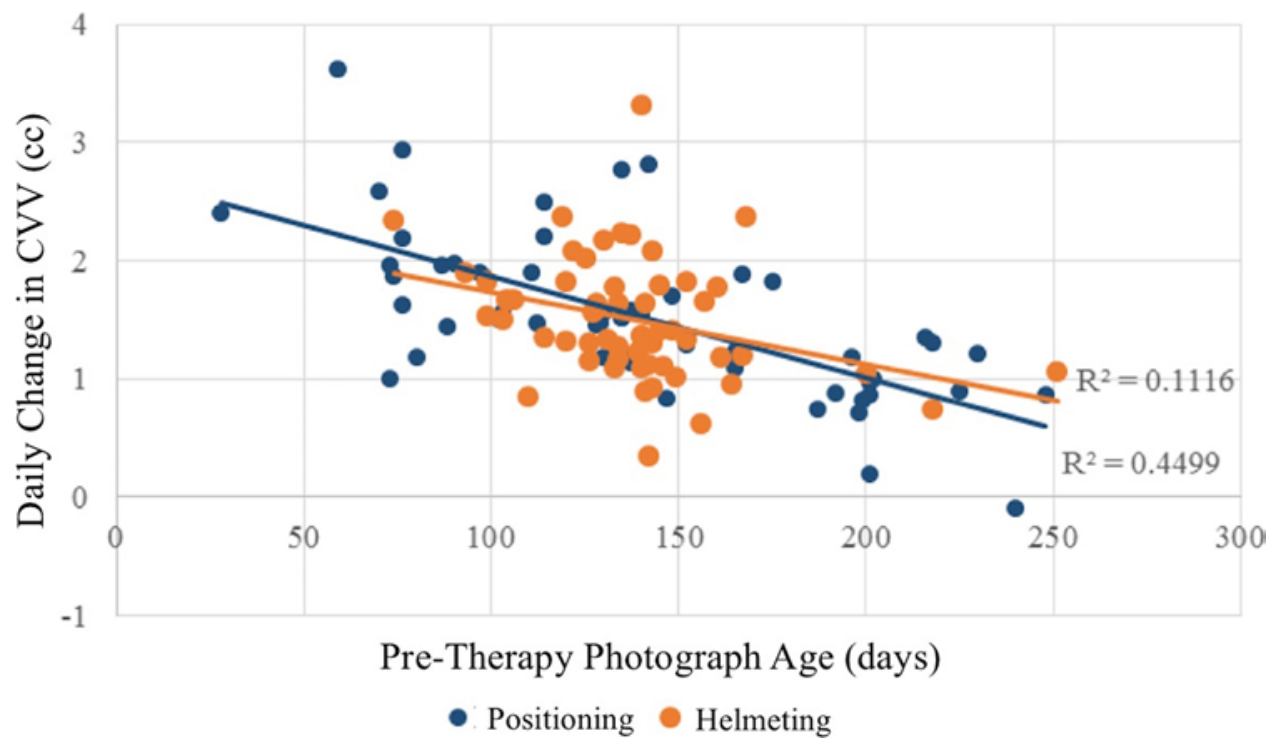

FIG. 5. The daily change in CVV (y axis) for each patient based on their age at the time when their pretherapy photograph was taken ( $x$ axis), showing a negative linear correlation in both study groups. Figure is available in color online only.

the infant is supine and more time in the prone position while awake. ${ }^{24,25}$ Helmeting therapy requires a custommade orthotic device for the infant that applies an external pressure to remold the skull when worn 22-23 hours per day. ${ }^{12,13,23}$ While these helmets are designed to allow space for growth and are regularly refitted, the possibility remains that they have the potential to restrict the infant's overall skull growth.

Cranial remolding helmets are also utilized with a similar helmeting protocol after endoscopic correction of craniosynostosis. The issue of cranial constriction has been voiced with regard to these craniosynostosis patients, ${ }^{6,9,27}$ and it has been articulated in the DPB literature..$^{12,15}$ The worry that using a helmet potentially constricts skull growth has not been proven in the literature. ${ }^{6,9,18}$

This study utilized two cranial volume measurements and head circumference to assess helmet constriction; we found no indication that the cranial orthosis limited growth in our study population. We chose to assess cranial constriction due to helmeting in the DPB population, as patients undergoing positioning therapy are well suited to forming a control group. In contrast, since surgical repair is standard treatment for all craniosynostosis patients, there is no similar control group for that cohort.

Volume measurements directly looking at the constrictive effects of helmets were not found in the literature on either DPB or craniosynostosis. In 1999 Kelly et al. were able to demonstrate normal growth trajectories in patients using helmets for DPB. This study was done before the availability of digital measuring tools and did not include a volume measurement. ${ }^{12}$ Collett et al., in 2012, found that total brain volume in patients with DPB was comparable to that in control patients before treatment, but they did not assess the change in volume with a posttreatment comparison. ${ }^{4}$ In 2013, Schweitzer et al. showed a normal increase in head circumference of DPB patients with helmet therapy compared to controls using $3 \mathrm{D}$ photographs. ${ }^{30}$ We were able to expand on this research by utilizing vol- ume measurements, as well as cranial circumference, and using patients with DPB as controls. Ghenbot et al., in 2015, compared the calvarial vault volume in patients with sagittal craniosynostosis before and after open surgery or endoscopic surgery with postoperative helmeting. They found no evidence of growth constriction in their 16 patients who had worn a helmet. ${ }^{6}$ However, this evidence was limited because of the potential impact of surgery on growth. Our study population allowed us to directly look at helmeting effects without this limitation.

The lack of a difference in cranial circumference or volume growth between the two study groups suggests that helmeting is not associated with cranial vault constriction in patients with DPB. Linear regression analyses found no significant difference between type of therapy and either circumference or volumetric measurements. Based on infant growth charts, we expected the patients' age to affect their growth rate. Pretherapy photograph age was a significant predictor of cranial growth, with younger patients averaging a greater change in volume and circumference measurements. These results extend previous reports on the safety of orthotic helmets for patients with DPB $^{12,15,30}$ and craniosynostosis. ${ }^{9,14,18}$ This study benefits

TABLE 2. Linear regression

\begin{tabular}{cccr}
\hline & $\begin{array}{c}\text { R-Squared } \\
\text { Value }\end{array}$ & b Value $(95 \% \mathrm{Cl})$ & $\mathrm{p}$ Value \\
\hline $\begin{array}{c}\text { Change in cubic vol, } \mathrm{ml} \\
\text { Pretherapy photograph } \\
\text { age, days }\end{array}$ & 0.26 & & $<0.001$ \\
\hline $\begin{array}{c}\text { Type of therapy } \\
\text { Change in CVV, ml }\end{array}$ & 0.36 & $15.9(-47.3$ to 79.1$)$ & 0.619 \\
\hline $\begin{array}{c}\text { Pretherapy photograph } \\
\text { age, days }\end{array}$ & & $-1.2(-1.5$ to -0.9$)$ & $<0.001$ \\
\hline \begin{tabular}{c} 
Type of therapy \\
\hline
\end{tabular} & & $5.9(-19.8$ to 31.6) & 0.650 \\
\hline
\end{tabular}


from its relatively large sample size and its use of detailed $3 \mathrm{D}$ photographs in the data collection process. The current study was powered to detect a fairly small change in volume $(35.2 \mathrm{ml})$ between the two therapy groups. The consistency of the results across different measurements also serves to strengthen the study conclusions.

Limitations of this study include its lack of randomization or blinding. Missing data and photographs reduced the number of patients from our database who met the inclusion criteria for this study. Despite the high ICC for reliability, there may still be human error introduced into the data through landmark placement on the photographs. We used Blender software to wrap the photographs containing holes to produce a more accurate volume measurement, but this method may still underestimate the true CVV. To minimize this error, we included a similar number of wrapped photographs in each therapy group (33 in the positioning group and 36 in the helmet group). The potential also exists for CVV measurement error from hair or skullcap bunching. The cubic volume measurement helps to alleviate the concern for this error. Compliance with the positioning or helmeting protocol and socioeconomic status were not able to be determined from our data set. Additionally, the data are from a single DPB clinic with a limited set of orthotic manufacturers and orthotists. Because of potential variability in helmeting protocols and manufacturing, this study could be strengthened by pooling data from multiple institutions. Future research in this area could also include long-term follow-up on patients' skull growth. Importantly, the applicability of these conclusions to patients fitted with helmets following surgical repair of craniosynostosis remains unsubstantiated.

\section{Conclusions}

Our study found no evidence that orthotic helmeting for skull reshaping in deformational plagiocephaly and/or brachycephaly was correlated with cranial vault constriction. This information may be used by health care providers and the patients' families to choose the appropriate therapy option for their child without worrying about longterm consequences associated with skull growth constriction.

\section{Acknowledgments}

Research reported in this publication was supported by funding to the Washington University Institute of Clinical and Translational Sciences (grant UL1 TR000448) from the National Center for Advancing Translational Sciences (NCATS) of the National Institutes of Health and Children's Discovery Institute. The content is solely the responsibility of the authors and does not necessarily represent the official view of the NIH.

Research was also supported by the Dean's Fellowship through the Office of Medical Student Research at Washington University in St. Louis School of Medicine.

\section{References}

1. Bialocerkowski AE, Vladusic SL, Howell SM: Conservative interventions for positional plagiocephaly: a systematic review. Dev Med Child Neurol 47:563-570, 2005

2. Bialocerkowski AE, Vladusic SL, Wei Ng C: Prevalence, risk factors, and natural history of positional plagiocephaly: a systematic review. Dev Med Child Neurol 50:577-586, 2008
3. Collett B, Breiger D, King D, Cunningham M, Speltz M: Neurodevelopmental implications of "deformational" plagiocephaly. J Dev Behav Pediatr 26:379-389, 2005

4. Collett BR, Aylward EH, Berg J, Davidoff C, Norden J, Cunningham ML, et al: Brain volume and shape in infants with deformational plagiocephaly. Childs Nerv Syst 28:10831090, 2012

5. De Bock F, Braun V, Renz-Polster H: Deformational plagiocephaly in normal infants: a systematic review of causes and hypotheses. Arch Dis Child 102:535-542, 2017

6. Ghenbot RG, Patel KB, Skolnick GB, Naidoo SD, Smyth MD, Woo AS: Effects of open and endoscopic surgery on skull growth and calvarial vault volumes in sagittal synostosis. J Craniofac Surg 26:161-164, 2015

7. Gump WC, Mutchnick IS, Moriarty TM: Complications associated with molding helmet therapy for positional plagiocephaly: a review. Neurosurg Focus 35(4):E3, 2013

8. Hutchison BL, Hutchison LA, Thompson JM, Mitchell EA: Plagiocephaly and brachycephaly in the first two years of life: a prospective cohort study. Pediatrics 114:970-980, 2004

9. Jimenez DF, Barone CM: Early treatment of coronal synostosis with endoscopy-assisted craniectomy and postoperative cranial orthosis therapy: 16-year experience. J Neurosurg Pediatr 12:207-219, 2013

10. Kane AA, Mitchell LE, Craven KP, Marsh JL: Observations on a recent increase in plagiocephaly without synostosis. Pediatrics 97:877-885, 1996

11. Kattwinkel J, Brooks J, Myerberg D: Positioning and SIDS. Pediatrics 89:1120-1126, 1992 (Erratum in Pediatrics 90:264, 1992)

12. Kelly KM, Littlefield TR, Pomatto JK, Manwaring KH, Beals SP: Cranial growth unrestricted during treatment of deformational plagiocephaly. Pediatr Neurosurg 30:193-199, 1999

13. Lam S, Pan IW, Strickland BA, Hadley C, Daniels B, Brookshier J, et al: Factors influencing outcomes of the treatment of positional plagiocephaly in infants: a 7-year experience. J Neurosurg Pediatr 19:273-281, 2017

14. Le MB, Patel K, Skolnick G, Naidoo S, Smyth M, Kane A, et al: Assessing long-term outcomes of open and endoscopic sagittal synostosis reconstruction using three-dimensional photography. J Craniofac Surg 25:573-576, 2014

15. Lee WT, Richards K, Redhed J, Papay FA: A pneumatic orthotic cranial molding helmet for correcting positional plagiocephaly. J Craniofac Surg 17:139-144, 2006

16. Lipira AB, Gordon S, Darvann TA, Hermann NV, Van Pelt AE, Naidoo SD, et al: Helmet versus active repositioning for plagiocephaly: a three-dimensional analysis. Pediatrics 126:e936-e945, 2010

17. Martiniuk AL, Vujovich-Dunn C, Park M, Yu W, Lucas BR: Plagiocephaly and developmental delay: a systematic review. J Dev Behav Pediatr 38:67-78, 2017

18. Marupudi NI, Sood S, Rozzelle A, Ham SD: Effect of molding helmets on intracranial pressure and head shape in nonsurgically treated sagittal craniosynostosis patients. J Neurosurg Pediatr 18:207-212, 2016

19. McGarry A, Dixon MT, Greig RJ, Hamilton DR, Sexton S, Smart H: Head shape measurement standards and cranial orthoses in the treatment of infants with deformational plagiocephaly. Dev Med Child Neurol 50:568-576, 2008

20. McKay DR, Davidge KM, Williams SK, Ellis LA, Chong DK, Teixeira RP, et al: Measuring cranial vault volume with three-dimensional photography: a method of measurement comparable to the gold standard. J Craniofac Surg 21:14191422,2010

21. McKinney CM, Cunningham ML, Holt VL, Leroux B, Starr JR: Characteristics of 2733 cases diagnosed with deformational plagiocephaly and changes in risk factors over time. Cleft Palate Craniofac J 45:208-216, 2008 
22. Moghaddam MB, Brown TM, Clausen A, DaSilva T, Ho E, Forrest CR: Outcome analysis after helmet therapy using 3D photogrammetry in patients with deformational plagiocephaly: the role of root mean square. J Plast Reconstr Aesthet Surg 67:159-165, 2014

23. Mortenson P, Steinbok P, Smith D: Deformational plagiocephaly and orthotic treatment: indications and limitations. Childs Nerv Syst 28:1407-1412, 2012

24. Naidoo SD, Skolnick GB, Patel KB, Woo AS, Cheng AL: Long-term outcomes in treatment of deformational plagiocephaly and brachycephaly using helmet therapy and repositioning: a longitudinal cohort study. Childs Nerv Syst 31:1547-1552, 2015

25. Paquereau J: Non-surgical management of posterior positional plagiocephaly: orthotics versus repositioning. Ann Phys Rehabil Med 56:231-249, 2013

26. Perlyn CA, Marsh JL, Vannier MW, Kane AA, Koppel P, Clark KW, et al: The craniofacial anomalies archive at St. Louis Children's Hospital: 20 years of craniofacial imaging experience. Plast Reconstr Surg 108:1862-1870, 2001

27. Proctor MR, Rogers GF: Helmets and synostosis. J Neurosurg Pediatr 9:680-682, 2012

28. Roberts SAG, Symonds JD, Chawla R, Toman E, Bishop J, Solanki GA: Positional plagiocephaly following ventriculoperitoneal shunting in neonates and infancy-how serious is it? Childs Nerv Syst 33:275-280, 2017

29. Schaaf H, Malik CY, Streckbein P, Pons-Kuehnemann J, Howaldt HP, Wilbrand JF: Three-dimensional photographic analysis of outcome after helmet treatment of a nonsynostotic cranial deformity. J Craniofac Surg 21:1677-1682, 2010

30. Schweitzer T, Böhm H, Linz C, Jager B, Gerstl L, Kunz F, et al: Three-dimensional analysis of positional plagiocephaly before and after molding helmet therapy in comparison to normal head growth. Childs Nerv Syst 29:1155-1161, 2013

31. Shweikeh F, Nuño M, Danielpour M, Krieger MD, Drazin D: Positional plagiocephaly: an analysis of the literature on the effectiveness of current guidelines. Neurosurg Focus 35(4):E1, 2013
32. Tamber MS, Nikas D, Beier A, Baird LC, Bauer DF, Durham S, et al: Congress of Neurological Surgeons systematic review and evidence-based guideline on the role of cranial molding orthosis (helmet) therapy for patients with positional plagiocephaly. Neurosurgery 79:E632-E633, 2016

33. van Wijk RM, van Vlimmeren LA, Groothuis-Oudshoorn CG, Van der Ploeg CP, IJzerman MJ, Boere-Boonekamp MM: Helmet therapy in infants with positional skull deformation: randomised controlled trial. BMJ 348:g2741, 2014

34. Xia JJ, Kennedy KA, Teichgraeber JF, Wu KQ, Baumgartner $\mathrm{JB}$, Gateno J: Nonsurgical treatment of deformational plagiocephaly: a systematic review. Arch Pediatr Adolesc Med 162:719-727, 2008

\section{Disclosures}

Dr. Patel is a consultant for Stryker CMF for education and product development. Drs. Naidoo and Patel are educational consultants for Hanger Clinic and Orthomerica.

\section{Author Contributions}

Conception and design: Naidoo, Peterson, Patel, Skolnick, Pfeifauf, Davidson. Acquisition of data: Peterson, Skolnick, Davidson. Analysis and interpretation of data: Peterson. Drafting the article: Peterson. Critically revising the article: Naidoo, Peterson, Patel, Skolnick, Pfeifauf, Smyth. Reviewed submitted version of manuscript: Naidoo, Peterson, Patel, Smyth. Approved the final version of the manuscript on behalf of all authors: Naidoo. Statistical analysis: Peterson, Skolnick. Study supervision: Naidoo, Patel, Skolnick.

\section{Correspondence}

Sybill D. Naidoo: Washington University School of Medicine, St. Louis,MO.naidoos@wustl.edu. 